\title{
El papel mediador de la capacitación docente en el manejo de la violencia escolar sobre el bienestar social de profesores
}

\author{
Macarena Morales, Verónica López, M. Ángeles Bilbao, Boris Villalobos, Denise Oyarzún, Dayana \\ Olavarría, Sebastián Ortiz, Claudia Carrasco y Paula Ascorra \\ Pontificia Universidad Católica de Valparaíso
}

\begin{abstract}
Resumen
La violencia escolar afecta negativamente, y el bienestar social escolar positivamente, la salud mental de los estudiantes y de sus profesores. En este estudio se analizó el efecto mediador de la necesidad de contar con capacitación en violencia escolar y de la magnitud de violencia escolar, en 397 profesores de escuelas básicas, sobre la asociación entre la frecuencia percibida de maltrato entre estudiantes y la coherencia social con que observan su contexto escolar. El modelo de mediación resultó significativo y mejoró el poder predictivo de la frecuencia de conductas de maltrato entre estudiantes sobre la coherencia social de la escuela. Sin embargo, es solo la percepción que los profesores tienen sobre su necesidad de capacitación en violencia escolar la variable que actúa como mediadora. Se discuten estos hallazgos en relación a la necesidad de fortalecer y mejorar los programas de capacitación y formación inicial y continua de los docentes.

Palabras clave: bienestar social, profesores, coherencia social, capacitación y violencia escolar.
\end{abstract}

\begin{abstract}
Students' and teachers' mental health is negatively affected by school violence, and positively affected by teachers' social wellbeing in school. We analyzed the mediating effect of teachers' perceived need to receive training in school violence prevention, as well as teachers' perceived magnitude of violence in their schools, on the negative association between the frequency of student maltreatment and teachers' perception of social coherence in their school. 397 elementary school teachers participated in this study. The mediating model theorized proved significant and improved the predictive power of the frequency of student maltreatment on teachers' social coherence in school. However, only teachers' perceptions of the need to receive teacher training on school violence had a mediating effect. Findings are discussed in terms of the need to strengthen and improve teacher training programs in school violence and school wellbeing, both for in-service as well as for teachers in formation.
\end{abstract}

Keywords: social wellbeing, teacher, social coherence, teacher training, school violence.

Correspondencia:

Av. Brasil 1290, Sausalito, Viña del Mar, Chile. E-mail: veronica.lopez @ucv.cl

Este estudio fue financiado por el Proyecto Financiamiento Basal CIAE FB0003, FONDEF IDEA CA12I10243 y FONDECYT 1140960. Agradecemos

la colaboración del Dr. Darío Páez en la revisión de este artículo. 


\section{Introducción}

La violencia en las escuelas es un problema que ha concitado la atención de investigadores y autoridades educacionales (López y Ascorra, 2012). Las consecuencias negativas que genera son llamativas por cuanto no solo afecta a quienes intimidan y a quienes reciben agresiones, sino que a toda la comunidad escolar, al deteriorar el clima escolar en general (Smith, 2006).

A nivel nacional, los resultados más recientes disponibles para Chile corresponden a la cantidad de denuncias que recibe la Superintendencia de Educación. La entidad ha informado que del total de denuncias, las referentes a maltrato físico y psicológico a los estudiantes corresponden a las más frecuentes, alcanzando un $37 \%$ en el período comprendido entre los meses de septiembre del año 2012 y septiembre del 2013 (Superintendencia de Educación, 2013). Luego de la Región Metropolitana-que reúne la cantidad más grande de habitantes-, la Región de Valparaíso concentra el mayor número de denuncias del país (Superintendencia de Educación, 2013). Un estudio realizado en esta última región indica que el $46 \%$ de los estudiantes refiere haber recibido intimidaciones por parte de sus compañeros en un año escolar, de los cuales el 9\% lo habría experimentado de manera persistente en el tiempo -"bastantes veces" o "casi todos los días"- (López, Morales y Ayala, 2009).

Investigadores del ámbito internacional sostienen que la mayor parte de los estudios sobre violencia en la escuela son realizados considerando a los estudiantes como informantes, recomendando que debiera indagarse más sistemáticamente en la percepción de los profesores al respecto (Zeira, Astor y Benbenishty, 2004; Espelage, Polanin y Low, 2014). La relevancia de ello radica en que los profesores son un agente importante en los procesos de socialización de los estudiantes en la escuela (Somech y Oplatka, 2009) y en que gran parte de los programas de prevención de la violencia consideran a los profesores en roles críticos, tales como en la creación de ambientes apropiados y en la promoción de cambios en las actitudes y comportamientos agresivos de sus estudiantes
(Ozer, 2006). No obstante, en una revisión que presentan Ávalos, Cavada, Pardo y Sotomayor (2010) se indica que los docentes se sienten principalmente afectados por la violencia en sus escuelas, en la medida que se sienten poco preparados para enfrentarla. También tendrían dificultades para analizar si su propia actuación y otros elementos contextuales exacerban las conductas violentas (ver también Bester y Du Plessis, 2010). El estudio de Arón y Milicic (2000) aporta con evidencias de esta situación, destacando que además incide en producir insatisfacción en el trabajo de los profesores.

Considerando estos antecedentes, este estudio exploró cómo incide la frecuencia de maltrato entre estudiantes percibida por profesores en la coherencia con que observan su contexto escolar. Ello, mediado por la percepción de magnitud del problema de la violencia en su escuela y por la necesidad de capacitación para enfrentarla.

\section{Percepción de coherencia social respecto de la escuela}

El bienestar subjetivo corresponde a las "valoraciones que la gente hace sobre su vida, los acontecimientos que suceden a ellos, sus cuerpos y mentes, y las circunstancias en que viven" (Diener, 2006, p. 400). Éste ha demostrado ser fundamental para mantener una salud mental positiva. El panorama de la investigación actual sobre la felicidad de las personas está protagonizado por tres perspectivas principales, a saber: el bienestar subjetivo (Diener, 2006), el bienestar psicológico (Ryff, 1998, en Vázquez y Hervás, 2008) y el bienestar social (Keyes, 1998). Estas tres propuestas ofrecen un aspecto que interesa reseñar: la relación del bienestar con el concepto de salud mental, el cual tiene como protagonista a un sujeto activo y socio-histórico (Blanco y Varela, 2007). En la Tabla 1 se presentan las dimensiones de las diferentes formas de conceptualizar el bienestar subjetivo en distintas tradiciones. 
Tabla 1.

Tradiciones en el estudio del bienestar subjetivo, psicológico y social.

\begin{tabular}{|c|c|c|}
\hline Bienestar subjetivo & Bienestar psicológico & Bienestar social \\
\hline $\begin{array}{l}\text { Satisfacción: juicio o } \\
\text { evaluación global de los } \\
\text { diversos aspectos que una } \\
\text { persona considera impor- } \\
\text { tantes en su vida. } \\
\text { Afecto positivo: resultado } \\
\text { de una experiencia emo- } \\
\text { cional placentera ante una } \\
\text { determinada situación vital. } \\
\text { Afecto negativo: resultado } \\
\text { de una experiencia emo- } \\
\text { cional negativa ante una } \\
\text { determinada situación vital. }\end{array}$ & $\begin{array}{l}\text { Auto-aceptación: sentirse bien consigo mismo, } \\
\text { actitudes positivas hacia uno mismo. } \\
\text { Relaciones positivas con los otros: mantenimiento } \\
\text { de relaciones estables y confiables. } \\
\text { Autonomía: capacidad para mantener sus convic- } \\
\text { ciones (autodeterminación), y su independencia y } \\
\text { autoridad personal. } \\
\text { Dominio del entorno: habilidad personal para } \\
\text { elegir o crear entornos favorables para satisfacer } \\
\text { los deseos y necesidades propias. } \\
\text { Objetivos vitales: metas que permitan dar sentido } \\
\text { a la vida. } \\
\text { Crecimiento personal: empeño por desarrollar las } \\
\text { potencialidades y seguir creciendo como persona. }\end{array}$ & $\begin{array}{l}\text { Integración social: sentimiento de pertenencia, } \\
\text { establecimiento de lazos sociales. } \\
\text { Aceptación social: confianza en los otros y } \\
\text { aceptación de los aspectos positivos y negativos } \\
\text { de nuestra propia vida. } \\
\text { Contribución social: sentimiento de utilidad, de } \\
\text { ser capaces de aportar algo a la sociedad en que } \\
\text { vivimos. Auto-eficacia. } \\
\text { Actualización social: Confianza en el futuro } \\
\text { de la sociedad, en su capacidad para producir } \\
\text { condiciones que favorezcan el bienestar. } \\
\text { Coherencia social: confianza en la capacidad } \\
\text { para comprender la dinámica y el funciona- } \\
\text { miento del mundo en el que nos ha tocado vivir. }\end{array}$ \\
\hline
\end{tabular}

Fuente: Elaboración propia en base a la literatura consultada.

Dentro de los determinantes del bienestar subjetivo se encuentran los aspectos sociales que marcan la vida de las personas (Keyes y López, 2005). Así, la presente investigación brinda un aporte relevante en el avance de la comprensión del bienestar subjetivo de los profesores, pues considera también el bienestar social (Keyes, 1998) contextualizado y situado en el mundo escolar, desde la perspectiva socialecológica (Bronfenbrenner, 1989; Espelage y Swearer, 2010).

El bienestar subjetivo tiene un componente hedónico nuclear, en términos de los aspectos emocionales y cognitivos que permiten a la persona evaluar su nivel de felicidad o bienestar presente y en términos generales (Diener, 2006), y un componente eudaimónico, relacionado con la evaluación personal del funcionamiento psicológico y social, que incluye la evaluación de la sociedad, las posibilidades de autodeterminación, la proyección al futuro y la construcción de sentido vital (Vásquez y Hervás, 2008; Keyes, 2013).

En los adultos, la relevancia de la evaluación del funcionamiento social y la percepción evaluativa de la sociedad en general constituyen pilares de la salud mental positiva (Keyes, 1998; Keyes y López, 2005; Keyes y Shapiro, 2004). El llamado bienestar social sería un pilar complementario al bienestar psicológico, que aporta a la construcción de una vida con sentido y propósito. En éste incide el tener relaciones significativas con otros, sentirse perteneciente de manera relevante a un mundo social percibido como inteligible y con potencial de desarrollo y futuro, al que la persona se siente ligada (Keyes, 2006).

El bienestar social ha sido definido como "la valoración que hacemos de las circunstancias y el funcionamiento dentro de la sociedad" (Keyes, 1998, p. 7). De esta forma, el bienestar social evalúa los aspectos interpersonales de la salud mental. Considerando estas dimensiones, la medición del bienestar social ha adquirido una creciente importancia en los últimos años, fundamentalmente debido a sus relaciones con la salud cívica y el capital social (Putnam, 2000) y muy especialmente con la salud mental desde una perspectiva biopsicosocial (Keyes y Shapiro, 2004; Keyes, 2013).

Las áreas que definen el bienestar social, según Keyes (1998), serían: integración social, aceptación social, contribución social, actualización social y coherencia social (Keyes, 1998; Keyes y Shapiro, 2004). La coherencia social se refiere al estado emocional de una persona, emergente a partir de las relaciones interpersonales que establece con su comunidad y de su valoración de la misma (Keyes, 1998). En este caso, la comunidad estudiada es la escuela donde el profesor trabaja.

Específicamente, la coherencia social respecto a la escuela se refiere a la percepción de comprensión de la dinámica de la comunidad, así como la sensación de poder influir en su funcionamiento (Ascorra, López, Bilbao, Correa, Guzmán, Moraga y Olavarría, 2014). La ocurrencia de actos violentos entre los estudiantes deteriora la sensación de seguridad, de claridad y de responsabilidad de los profesores frente al problema (Bester y Du Plessis, 2010; Arón y Milicic, 2000), lo que podría afectar su percepción de coherencia en el plano interpersonal, en el contexto de la escuela.

\section{Maltrato entre estudiantes}

El maltrato entre los estudiantes puede entenderse como una forma de comportamiento de riesgo que se distingue por la intención de causar daño o menoscabo (Steffgen, 2009). En este estudio se consideran comportamientos de 
gravedad el uso de armas u objetos para herir, y agresiones menos graves como empujar a un compañero (Somech y Oplatka, 2009). También incluye acciones que produzcan daño emocional o psicológico, como el proferir insultos, el hablar mal de otros y las amenazas verbales (Blaya, 2003).

La investigación sobre maltrato escolar, comúnmente llamado bullying, indica que tiene consecuencias directas sobre la salud mental de las personas afectadas (víctimas y agresores), así como sobre el grupo de testigos y la comunidad escolar. Además, afecta negativamente el clima escolar del establecimiento (Smith, 2006).

\section{Percepción de magnitud de la violencia escolar}

La magnitud de la violencia escolar se refiere a la gravedad que los profesores perciben sobre la violencia que ocurre en su escuela (Zeira, Astor y Benbenishty, 2004). Desde la perspectiva de los profesores, se observa una tendencia en Chile a considerar que la violencia escolar es un problema grande (Ministerio de Educación, Organización de Naciones Unidas e IDEA, 2005; Universidad Alberto Hurtado, Ministerio del Interior y Ministerio de Educación, 2006). Sin embargo, hay antecedentes que otorgan respaldo a la idea de que la percepción global de la violencia escolar no necesariamente se condice con los niveles comportamentales de maltrato entre estudiantes u otras forma de agresión. Generalmente, la percepción global de violencia escolar (i.e. preguntas del tipo ¿cuán importante/grave es la violencia en su escuela?) es mayor a la frecuencia de conductas o comportamientos de maltrato en la escuela (i.e. preguntas del tipo ¿cuántas veces, en el último mes, han ocurrido las siguientes situaciones?) (Benbenishty y Astor, 2005).

De acuerdo a Sebastião (2013), es generalmente aceptado que la violencia en las escuelas es un problema inadmisible; a su vez, debe considerarse que a nivel cultural hay formas de violencia aceptadas como normales de ejercer por parte de ciertos grupos, en razón de su edad, nivel socioeconómico y género, entre otras categorías. Por ejemplo, la agresión física que ocurre entre estudiantes hombres sería percibida como menos grave (Bester y Du Plessis, 2010), en comparación de si ocurre hacia o entre mujeres (Velásquez, Bruce, Saldarriaga, López y Bukowski, 2010). Esto podría afectar, por una parte, la incidencia de la frecuencia de maltratos entre estudiantes en la magnitud percibida del problema. Por otra parte, ello podría afectar en el nivel en que la magnitud de violencia escolar incide en la coherencia social percibida en la escuela. De allí que hipotetizamos que la percepción que los profesores tienen acerca de la magnitud de la violencia escolar podría estar actuando como mediadora de la relación negativa entre su percepción de violencia escolar medida como conductas específicas de los estudiantes, y su percepción subjetiva de coherencia social en la escuela.

\section{Capacitación para enfrentar la violencia escolar}

Los profesores muchas veces refieren la necesidad de entrenarse para prevenir y enfrentar la violencia escolar. Como ya se ha descrito, la literatura señala que los profesores tienden a sentirse poco preparados para tratar la violencia entre sus estudiantes, afectando en su nivel de satisfacción en el trabajo (Arón y Milicic, 2000) y en sentirse pertinentes para abordarla (Bester y Du Plessis, 2010). Cornejo (2007) sostiene que los problemas de disciplina en la escuela son uno de los factores que se asocian al deterioro de la salud mental de los docentes, quienes en Chile presentan alarmantes cifras de trastornos ansiosos y depresión mayor (Cornejo, 2009).

La investigación de Zeira et al. (2004), realizada con 1.521 profesores en Israel, arrojó que la mayoría de ellos reconoció necesitar capacitación frente a la violencia escolar (29\% indicó necesitarla en gran medida y un $25 \%$ moderadamente). Ello, a pesar de que solo un $10.3 \%$ consideró que la violencia en su escuela era un problema grande y un $26.7 \%$ lo definió como un problema pequeño o nulo. Los autores recomendaron indagar más en la relación entre estas variables. Sela-Shayovitz (2009) reportó el análisis de una encuesta aplicada a 147 profesores. La autora encontró diferencias significativas entre los docentes que han realizado entrenamiento para enfrentar la violencia escolar, frente a aquellos que no lo han realizado, indicando que los primeros se perciben más eficaces en su trato con la violencia que ocurre en su escuela que los segundos: $\mathrm{t}(1,147)=-4.48$, $\mathrm{p}<.0001(\mathrm{M}=3.50, \mathrm{DT}=0.71$, versus $\mathrm{M}=2.89, \mathrm{DT}=$ 0.88 , respectivamente).

Estos antecedentes permiten proyectar que a mayor frecuencia de maltratos percibidos entre estudiantes, aumentará la necesidad de capacitación para enfrentar la violencia. Además, que a mayor necesidad de capacitación para enfrentar la violencia, menor será la coherencia social en la escuela para los profesores. De allí que en este estudio hipotetizamos que el que los docentes sientan la necesidad de recibir capacitación para abordar la violencia escolar, podría estar actuando como mediador de la relación negativa entre la violencia escolar y la coherencia social de los profesores en sus escuelas.

Considerando estos antecedentes, este estudio buscó indagar, mediante un modelo de mediación, cómo incide 
la frecuencia de maltrato entre estudiantes percibida por profesores en la coherencia con que observan su contexto escolar. Ello, mediado por la percepción de magnitud del problema de la violencia en su escuela y por la necesidad de capacitación para enfrentarla.

\section{Metodología}

El objetivo del estudio fue conocer si la gravedad de la violencia escolar y la necesidad de capacitación explican la varianza en la coherencia social que los profesores perciben en su comunidad escolar, por sobre el impacto que tiene en ello la frecuencia de maltrato entre los estudiantes. Una hipótesis es que la frecuencia de maltrato entre alumnos tiene una relación negativa con la coherencia social. Dados los antecedentes teóricos derivados de la investigación, presentados anteriormente, se esperaba que esta relación estuviera mediada por la necesidad de capacitación para enfrentar la violencia escolar y por la magnitud de la violencia percibida. Las hipótesis del estudio se organizan en el modelo teórico graficado en la Figura 1.

\section{Participantes}

Participaron 397 profesores (282 mujeres y 105 hombres) que trabajaban en enseñanza básica, provenientes de 44 establecimientos educativos de la ciudad de Valparaíso, Chile. Contestaron una encuesta online de un proyecto de monitoreo de la convivencia escolar ${ }^{1}$, ejecutado en la totalidad de escuelas municipales con enseñanza básica de Valparaíso, Chile. Del total de colegios, 43 corresponden a áreas geográficas urbanas y uno a escuela rural. El índice de vulnerabilidad de los establecimientos de la comuna alcanza el $70 \%$.

Del total de participantes, el $22.7 \%(n=88)$ de los profesores tenía entre uno y tres años de experiencia docente, el $32 \%(n=124)$ entre cuatro y diez años, el $14.2 \%(n=$ $55)$ entre once y veinte años y el $31 \%(n=120)$ contaba con más de veinte años. Respecto de la permanencia en su puesto de trabajo actual, un $61 \%(n=236)$ había trabajado en la misma escuela entre uno y cinco años, y un $28.4(n=$ 110) había trabajado en la misma escuela entre seis y veinte años. Asimismo, el 10.6\% $(n=41)$ de profesores estaba trabajando hace más de veinte años en la misma escuela.

\footnotetext{
1 Proyecto FONDEF-IDeA CA12i10243, ejecutado por el Programa de Apoyo a la Convivencia Escolar de la PUCV, www.paces.cl.
}

La selección de la muestra de participantes fue no probabilística e intencionada, puesto que se escogieron a los profesores de enseñanza básica presentes en las escuelas el día de aplicación de la encuesta, pertenecientes al 100\% de las escuelas municipales de la comuna que imparten dicho nivel educacional.

\section{Instrumentos}

Las variables medidas formaron parte de un cuestionario online contestado por los profesores. El cuestionario está constituido principalmente por una adaptación del instrumento propuesto por Zeira et al. (2004). La adaptación consistió en una traducción de los ítems y alternativas de respuesta al español de Chile. Consiste en un auto y hetero-reporte, anónimo, con respuestas en intervalos de frecuencias. Las variables que este estudio consideró fueron:

Conductas de maltrato entre estudiantes. Este instrumento corresponde a un hetero-reporte del profesor acerca de su percepción de frecuencia de conductas de maltrato entre estudiantes. Comprende cuatro ítems que indagan en si los estudiantes se involucran en peleas, robos, amenazas e intimidación verbal y porte de armas blancas en la escuela. Las opciones de respuesta fueron: $1=$ "Nunca", $2=$ "1 ó 2 veces", 3 = "Más de 3 veces" y 99 = "No sé/No quiero contestar".

Magnitud de la violencia escolar percibida. Esta variable fue medida a través de un ítem (¿Cuál es la magnitud del problema de violencia escolar en tu escuela?) con seis opciones de respuesta, correspondiendo: $1=$ "No es un problema", 2 = "Es un pequeño problema", 3 = "Es un problema mediano", 4 = "Es un problema grande", 5 = "Es un problema muy grande" y 99 = "No sé/No quiero contestar".

Necesidad de capacitación para ayudar a prevenir y reducir la violencia escolar. Esta variable fue medida a través de un ítem (¿Siente usted que necesita capacitación para ayudar a prevenir y reducir la violencia escolar?) con seis opciones de respuesta, correspondiendo: 1 = "Para nada, en absoluto", 2 = "Algo", 3 = "En cierta medida", 4 $=$ "En gran medida", 5 = "A un grado muy grande" y $99=$ "No sé/No quiero contestar".

Se incluyó en el cuestionario también la Escala de Bienestar Social de Keyes (1998), adaptada al contexto escolar por Ascorra et al. (2014). Para este estudio se utilizó la siguiente subescala:

Coherencia social en la escuela $(\mathrm{a}=.76)$. Se compone de cuatro ítems referentes a comprender la dinámica social de la escuela. Las opciones de respuesta fueron 1 = "Muy 
de acuerdo", 2 = "De acuerdo", 3 = "En desacuerdo", 4 = "Muy en desacuerdo" y 99 = "No sé/No quiero contestar".

\section{Procedimiento}

Previo a la aplicación de la encuesta, se obtuvo la autorización de cada establecimiento y el consentimiento de los profesores. Con anticipación a la aplicación de la encuesta, cada establecimiento participante en el proyecto de investigación confirmó la nómina de profesores que enseñaban en clases de $4^{\circ}$ a $8^{\circ}$ año básico. Se generó una clave única para cada profesor, la cual fue proporcionada por un encuestador del equipo ejecutor del proyecto o por un encargado del interior de su establecimiento, según se acordara con cada dirección de escuela. Esta clave aseguraba que cada docente ingresara una sola vez al sistema a contestar su encuesta, y por parte del equipo investigador se aseguraba la confidencialidad de la misma. Los cuestionarios fueron contestados individualmente por los profesores en una plataforma virtual (www.paces.cl), haciendo uso de sus computadores personales o de los de la sala de computación de su establecimiento. En la mayoría de las escuelas, la encuesta se aplicó durante la hora de reflexión pedagógica de forma masiva. En el resto de las escuelas, el encargado de convivencia escolar entregó las claves de manera personal a cada docente, cautelando que contestaran la encuesta a la brevedad. Cada profesor firmó un consentimiento informado, en el que se comprometía la confidencialidad y el anonimato de sus respuestas.

\section{Análisis de datos}

Para contrastar el modelo de mediación propuesto teóricamente, se condujo un análisis de mediación múltiple con dos mediadores, utilizando el macro de SPSS para efectos de mediación múltiple (Preacher y Hayes, 2008) con el método de bootstrapping. Los coeficientes se estimaron desde 5000 muestras bootstrap.

\section{Resultados}

Antes de aplicar el modelo de mediación, se verificó el cumplimiento de los supuestos de la regresión múltiple. También se testeó la relación entre las variables a introducir en el modelo. Estos resultados preliminares indicaron una asociación significativa entre todas las variables (ver Tabla 2), para la muestra bajo estudio. Los valores fluctuaron entre -0.19 y 0.78 . La frecuencia de maltrato entre estudiantes mostró una correlación alta y positiva con la necesidad de capacitación y la magnitud de la violencia escolar percibida. En tanto, las correlaciones fueron negativas entre la frecuencia de maltrato y la coherencia social. También fueron negativas entre la necesidad de capacitación y la magnitud de la violencia escolar percibida, con la coherencia social.

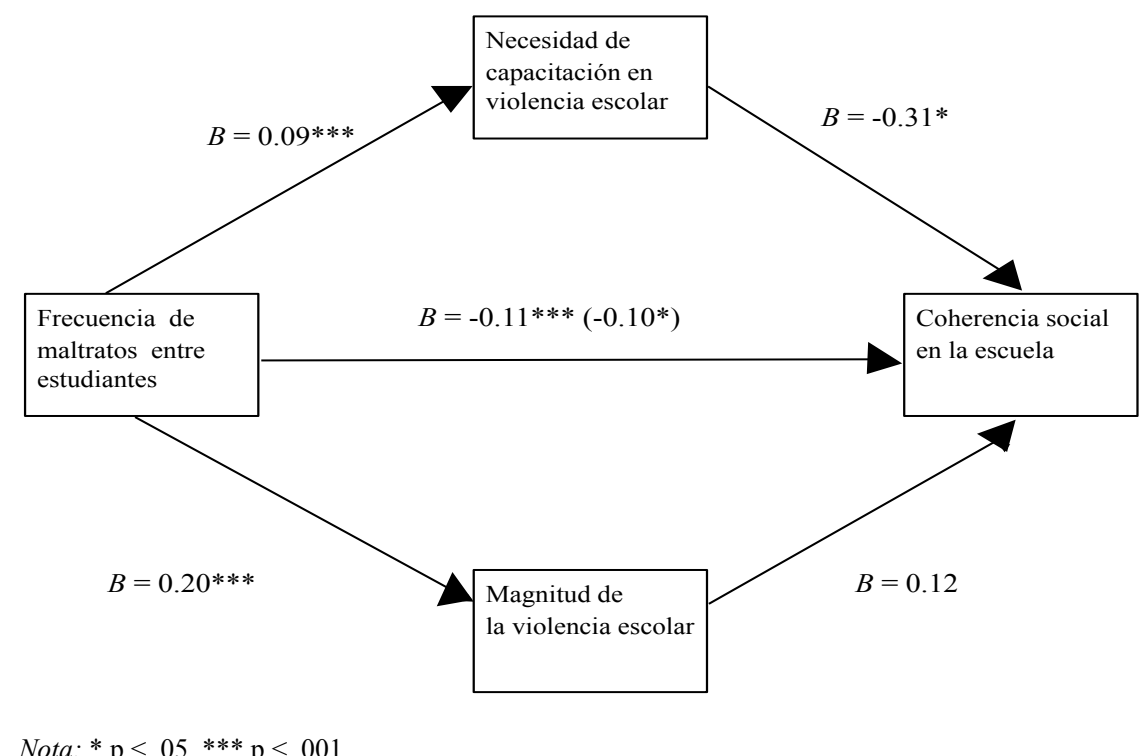

Figura 1.

Modelo de mediación propuesto y contrastado. 
Tabla 2.

Correlaciones bivariadas entre las variables mediadoras, dependiente y predictora.

\begin{tabular}{lllll}
\hline & 1 & 2 & 3 & 4 \\
\hline 1. Maltrato entre estudiantes & - & & & \\
2. Coherencia social & $-.19^{* *}$ & - & & \\
3. Necesidad de capacitación & $.39^{* *}$ & $-.18^{* *}$ & - & \\
$\begin{array}{l}\text { 4. Magnitud de la violencia } \\
\text { escolar }\end{array}$ & $.78^{* *}$ & $-.14^{* *}$ & $.49^{* *}$ & - \\
\hline
\end{tabular}

Nota: $\mathrm{N}=397, * * p<.01$ (unilateral).

La Figura 1 representa el modelo de mediación propuesto, incluyendo los efectos directos, indirectos y totales. El único efecto que no resultó significativo fue el de la magnitud de la violencia escolar sobre la coherencia social en la escuela.

El efecto de la frecuencia de maltrato entre estudiantes sobre la coherencia social aumenta su magnitud al ingresar las variables mediadoras (desde $B=-0.10, p=0.03$ a $B=$ $-0.11, p<0.00)$. El modelo en su conjunto explica un $5.1 \%$ de la varianza de la coherencia social de los profesores en la escuela.

La frecuencia de maltratos entre estudiantes arrojó coeficientes positivos sobre la magnitud de violencia percibida y sobre la necesidad de capacitación para enfrentar la violencia escolar. La magnitud de la violencia escolar no tuvo coeficiente significativo sobre la coherencia social, en tanto que la necesidad de capacitación sí, siendo este negativo.

\section{Discusión}

Los resultados indican que el modelo de mediación resultó significativo y mejoró el poder predictivo de la variable dependiente (frecuencia de conductas de maltrato entre estudiantes) sobre la variable predictora (coherencia social de la escuela). Sin embargo, es solo la percepción que los profesores tienen sobre su necesidad de capacitación en violencia escolar la variable que estaría actuando como mediadora de la relación entre maltrato percibido entre estudiantes y el nivel de coherencia social en la escuela, componente propio del bienestar social escolar.

Los resultados antes expuestos indican que la violencia en la escuela incide en deteriorar la coherencia que los profesores perciben en su comunidad escolar. Esto es concordante con lo arrojado por los análisis cualitativos de Bester y Du Plessis (2010) y de Arón y Milicic (2000), respecto a que el maltrato entre los estudiantes es un elemento que afecta la sensación de pertinencia y de bienestar de los profesores en su ámbito laboral.
La violencia entre los estudiantes también afecta su magnitud percibida por los profesores, agravándola. Esto puede ser interpretado en relación a que las situaciones de violencia que se dan en las escuelas son sopesadas por los docentes, generando en ellos una opinión de que no es una situación normal en el estudiantado. Sebastião (2013) señala que algunas formas de violencia en la escuela tienden a normalizarse en relación a la edad, género o clase social, lo que en esta muestra no sucedería en los estudiantes, quienes son de enseñanza básica y de un contexto social en general vulnerable.

Los hallazgos de este estudio sugieren que la magnitud con que los profesores perciben el problema de la violencia no tiene un efecto significativo sobre la coherencia social, lo que puede decir que sea cual sea la gravedad del maltrato no afecta en cómo los profesores comprenden sus interacciones en la escuela. Este resultado puede considerarse positivo, por cuanto sugiere que los profesores tendrían conciencia de que cualquier nivel de violencia entre sus estudiantes representa un problema, lo cual puede constituir un primer nivel de sensibilización para avanzar en tareas de prevención. Pero también cabe pensar que la alta correlación entre la percepción de frecuencia de conductas de maltrato entre estudiantes y la percepción de la magnitud del problema hallada en este estudio $(r=.78)$ expliquen el bajo poder predictor de la variable percepción de magnitud del problema como mediadora.

La frecuencia de maltrato entre estudiantes aumenta la necesidad de capacitación para abordar el problema, lo cual aporta información a las interrogantes planteadas por Zeira et al. (2004) acerca de esta última variable. A mayor necesidad de capacitación, decrece significativamente la coherencia social de los profesores, lo que llama a valorar la importancia de brindar formación continua para enfrentar el problema. Esta recomendación se robustece de los antecedentes coherentes con este hallazgo, como los presentados por Ávalos et al. (2010) y Sela-Shayovitz (2009), pues la formación inicial y continua permitiría mejorar la respuesta frente a la violencia en la escuela, y además la percepción docente acerca de que la escuela es comprensible y modificable.

Una implicancia directa de los hallazgos de este estudio es el papel fortalecedor que la capacitación docente en violencia escolar y convivencia escolar podría tener, tanto sobre la disminución del nivel de maltrato percibido entre estudiantes, como sobre la coherencia social percibida de la escuela. Ello, toda vez que dicha capacitación sea comprendida como un aporte significativo y disminuya efectivamente la percepción de "necesitar más capacitación", lo que podría a su vez aumentar la percepción de autoeficacia en el manejo 
de la violencia escolar. Al respecto, la literatura sobre formación docente ha sido insistente en señalar que no todas las formas de capacitación docente resultan efectivas. Al respecto, una metodología de creciente interés para la formación docente en violencia y convivencia escolar, ha sido el uso de propuestas de resolución de problemas, tales como el uso de incidentes críticos (Nail, Gajardo y Muñoz, 2012).

Otra implicancia corresponde a la relevancia de proteger el bienestar social del profesor. Como enfatiza la literatura sobre bienestar social, la capacidad para comprender la dinámica y el funcionamiento del mundo en el que toca vivir -en este caso, la escuela- es un elemento que contribuye a construir una vida con sentido y propósito. Además, desde el ámbito de la clínica escolar, cuidar y prevenir la salud mental de los profesores repercute en resguardar el ambiente y la salud mental de los propios estudiantes (Milicic, Mena, López y Justiniano, 2008).

En cuanto a las limitaciones de este estudio, los resultados deben considerarse cautelosamente, pues no se utilizó un muestreo representativo de la población. Se trabajó con profesores solo de enseñanza básica, de dependencia municipal y de una comuna de Chile, lo que deja planteado el desafío de avanzar enriqueciendo la muestra, para poder generalizar resultados y orientar decisiones a nivel nacional. Dentro de las limitaciones también debe considerarse que el instrumento de medición de la magnitud de violencia percibida y de la necesidad de capacitación correspondió a un ítem dentro de una encuesta, lo que afecta su confiabilidad estadística. En relación a ello, futuros estudios pueden encaminarse a desarrollar la medición de estas dimensiones.

\section{Referencias}

Arón, A. M. y Milicic, N. (2000). Desgaste profesional de los profesores y clima social escolar. Revista Latinoamericana de Psicología, 32(3), 447-466.

Ascorra, P., López, V., Bilbao, M. Á., Correa, T., Guzmán, J., Moraga, V. y Olavarría, D. (2014). Relación entre el bienestar social de Profesores y el Nivel de Autonomía y Tamaño de Escuelas Municipalizadas Chilenas. Terapia Psicológica, 32(2), 121-132.

Ávalos, B., Cavada, P., Pardo, M. y Sotomayor, C. (2010). La profesión docente: temas y discusiones en la literatura internacional. Estudios Pedagógicos, 36(1), pp. 253-263.

Bester, S. y Du Plessis, A. (2010). Exploring a secondary school educator's experiences of school violence: a case study. South African Journal of Education, 30(2), 203-229.

Benbenishty, R. y Astor, R. A. (2005). School violence in context: Culture, neighborhood, family, school, and gender. New York: Oxford University Press.

Blanco, A. y Varela, S. (2007). Los fundamentos de la intervención psicosocial. En: A. Blanco y J. Rodríguez Marín (Coords.). Intervención Psicosocial. Madrid: Pearson, Prentice Hall.
Blaya, C. (2003). School violence and the professional socialisation of teachers: The lessons of comparatism. Journal of Educational Administration, 41(6), 650-668.

Bronfenbrenner, U. (1989). Ecological systems theory. Annals of Child Development. 6, 187-249.

Chile, Ministerio de Educación, Organización de las Naciones Unidas para la Educación, la Ciencia y la Cultura e Instituto de Evaluación y Asesoramiento Educativo (2005). Estudio Nacional de Convivencia Escolar: la opinión de estudiantes y docentes 2005.

Chile, Ministerio del Interior, Ministerio de Educación y Universidad Alberto Hurtado (2006). $1^{\circ}$ Encuesta Nacional de Violencia en el Ámbito Escolar. Informe Encuesta Profesores.

Cornejo, R. (2007). Bienestar/malestar docente y condiciones de trabajo en profesores de enseñanza media de Santiago. Santiago: Ministerio de Educación de Chile, Fondo Nacional de Investigación y Desarrollo en Educación - FONIDE.

Cornejo, R. (2008). Salud laboral docente y condiciones de trabajo. Revista Docencia, 35, 77-85.

Diener, E. (2006). Guidelines for national indicators of subjective wellbeing and ill-being. Journal of Happiness Studies, 7, 397-404.

Espelage, D. L., Polanin, J. R. y Low, S. K. (2014). Teacher and staff perceptions of school environment as predictors of student aggression, victimization, and willingness to intervene in bullying situations. School Psychology Quarterly, 29(3), 287-305.

Espelage, D. L. y Swearer, S. M. (Eds.) (2004). Bullying in American schools: A social-ecological perspective on prevention and intervention. Taylor y Francis e-library.

Keyes, C. L. M. (1998). Social well-being. Social Psychology Quarterly, 61(2), 121-140.

Keyes, C. L. M. (2006). Subjective Well-Being in Mental Health and Human Development Research Worldwide: An Introduction. Social Indicators Research, 77, 1-10.

Keyes, C. L. M. (Ed.) (2013). Mental Well-Being. International Contributions to the Study of Positive Mental Health. Springer: GA, USA.

Keyes, C. L. M. y López, S. J. (2005). Toward a science of mental health. En C. R. Snyder y S. J. López (Eds.). Handbook of Positive Psychology (pp.45-59). Oxford: Oxford University Press.

Keyes, C.L.M. y Shapiro, A. (2004). Social Well-Being in the United States: A Descriptive Epidemiology. In O. G. Brim, C. D. Ryff y R. C. Kessler (Eds.). How Healthy Are We?: A National Study of Well-Being at Midlife (pp. 350-372). Chicago: University of Chicago Press. López, V., Morales, M. y Ayala, Á. (2009). Maltrato entre pares: conductas de intimidación y victimización en escolares chilenos. Revista de Psicologia, 27(2), 243-286.

López, V. y Ascorra, P. (2012). Miradas de la violencia en el espacio social de la escuela. Psicoperspectivas, 11(2), 1-7.

Milicic, N., Mena, M. I., López, V. y Justiniano, B. (2008). Clínica escolar: Prevención y atención en redes. Santiago: Ediciones Universidad Católica de Chile.

Nail, O., Gajardo, J. y Muñoz, M. (2012). La técnica de análisis de incidentes críticos: Una herramienta para la reflexión sobre prácticas docentes en convivencia escolar. Psicoperspectivas, 11(2), 56-76.

Ozer, E. J. (2006). Contextual effects in school-based violence prevention programs: A conceptual framework and empirical review. Journal of Primary Prevention, 27(3), 315-340.

Preacher, K. J. y Hayes, A. F. (2008). Asymptotic and resampling strategies for assessing and comparing indirect effects in multiple mediator models. Behavior Research Methods, 40, 879-891.

Putnam, R. (2000). Bowling Alone: The Collapse and Revival of American Community. New York: Simon and Schuster.

Sebastião, J. (2013). Violência na escola, processos de socialização e formas de regulação. Sociologia, problemas e práticas, 71, 23-37.

Sela-Shayovitz, R. (2009). Dealing with school violence: The effect of school violence prevention training on teachers' perceived self-efficacy in dealing with violent events. Teaching and Teacher Education, 25(8), 1061-1066

Smith, P. K. (2006). School violence dissected: An Israeli view. Human Development, 49, 248-252. 
Somech, A. y Oplatka, I. (2009). Coping with school violence through the lens of teachers' role breadth: The impact of participative management and job autonomy. Educational Administration Quarterly, 45(3), 424-449.

Steffgen, G. (2009). Deviant behaviour and violence in Luxembourg Schools. International Journal of Violence in Schools, 10, 54-70.

Superintendencia de Educación (2013). Informe de denuncias, Superintendencia de Educación Nacional. Total Nacional. Santiago: Gobierno de Chile.

Vázquez, C. y Hervás, G. (2008). Psicología Positiva Aplicada. Bilbao: Desclee de Brower.

Velásquez, A. M., Bruce, J. S., Saldarriaga, L. M., López, L. S. y Bukowski, W. M. (2010). Context-dependent victimization and aggression: Differences between all-girl and mixed-sex schools. Merrill-Palmer Quarterly, 56(3), 283-302.

Zeira, A., Astor, R. A. y Benbenishty, R. (2004). School Violence in Israel: Perceptions of Homeroom Teachers. School Psychology International, 25(2), 149-166. 
